\title{
Aggressive or conservative management in extradural hematomas in children - a challenging neurosurgical choice
}

\author{
A. Tascu' ${ }^{1}$, C. Pascal ${ }^{2}$, St.M. Iencean ${ }^{3}$, M.R. Gorgan ${ }^{1}$ \\ "'Carol Davila" University of Medicine and Pharmacy Bucharest \\ ${ }^{2}$ Neurosurgery, "Bagdasar Arseni" Hospital, Bucharest \\ 3"Grigore T. Popa" University of Medicine and Pharmacy Iasi
}

\begin{abstract}
EDH) in children appear as a consequence of head trauma. Although emergency surgical intervention was the classical neurosurgical treatment for $\mathrm{EDH}$, lately there has been observed a tendency to replace operation by conservative management, whenever the neurological status and imaging appearance allows it. The aim of this article is to present our experience in treating EDH in children 0-3 years old and to establish a management protocol for EDH in infants, by evaluating the clinical and neuroimaging status, of both surgically and conservatively treated patients, from hospital admission to discharge. Retrospective study includes 52 patients diagnosed with an extradural hematoma, admitted in the First Neurosurgery Department of the Clinical Hospital 'Bagdasar-Arseni' in Bucharest, from January 2004 to December 2013. The patients were identified by diagnosis from the clinic's database; clinical and imaging data was extracted from the patient's individual records and crosschecked with the operating protocols. Cerebral CT scan was the preferred imaging investigation for diagnosis. Our study includes 52 patients ( 26 boys and 26 girls), with a mean age of 14.5 months (range 6 weeks - 3 years old). 25 patients were surgically treated, while the other 27 received symptomatic medication and were monitored clinically and by imaging exams. The most frequent clinical manifestations were intracranial hypertension (21 patients) and psychomotor agitation (19 patients). The traumatic mechanisms were: accidental falling (38 patients), blunt head trauma (3 patients), road accident ( 2 patients), unspecified (8 patients) other causes (1 patient). Based on the Glasgow Coma Scale classification of TBI, 39 patients suffered a mild TBI, 7 a moderate TBI and 6 patients suffered a severe TBI. Most of the patients had a good recovery; there was a total of two deaths. The most common location for the EDHs was parietal (20 patients) and temporal-parietal (11 patients). Both surgical treatment and conservative management of EDH have a good clinical outcome. Clinical and neuroimaging evaluation at admission/reevaluation plays an imperative role in deciding the appropriate therapeutic attitude for each patient.
\end{abstract}

Key words: conservative therapy, extradural hematomas in children, surgical therapy. 


\section{Introduction}

An extradural hematoma (EDH) is a blood clot that develops between the dura mater and the skull, it usually has a biconvex shape and it is found in $2.5 \%$ to $5 \%$ of head injured patients(1). Head trauma in children $0-3$ years old is completely different compared with adults - 'children are not young adults' (2). EDHs are not a common finding in children presenting with head trauma, being diagnosed in only $1-3 \%$ of these patients (3-5). The most frequent mechanisms for head trauma in children are either accidental falling of blunt head trauma (7-9). In $73 \%$ of the EDH the bleeding source is the middle meningeal artery or vein, usually associated to a temporal bone fracture (10). EDHs may also be of venous origin as the result of tearing of venous dural sinuses, emissary veins, or venous lakes within the dura mater. Most traumatic venous EDHs occur in children, and most are not associated with a skull fracture (10). EDHs are often located temporal-parietal, due to the easily detachment of the dura from the bone structure - dural detachment areas of GerardMarchand.

The specific management of epidural hematomas was not thoroughly established and proposed in class I or II evidence-based guidelines, and their treatment is based on clinical and brain imaging criteria such as GCS score, pupillary anomalies, volume, thickness and mass effect detected on CT scan, as well as the neurosurgeon's personal experience. Our study describes the results on 52 pediatric patients (age 0-3 yo) treated surgically or conservative.

\section{Methods}

Inclusion and exclusion criteria. The study includes 52 patients diagnosed with an extradural hematoma, admitted in the First Neurosurgery Department of the Clinical Hospital 'Bagdasar-Arseni' in Bucharest, from January 2004 to December 2013. There were 72 patients admitted with a diagnosis of epidural hematoma, but 20 patients were not included in the study due to the incomplete data in the patients files.

Study design and database. The present study is a retrospective analysis of 52 patients aged 0-3 years old, treated in our department during the period from January 2004 to December 2013 for a EDH. The patients were identified by diagnosis (ICD 10 - S06.4: Epidural hemorrhage) from the clinic's computerized database; clinical and imaging data was extracted from the patient's individual records and crosschecked with the operating protocols. The following data was analyzed: demographic data - age, sex; case history data regarding the mechanism of the TBI and the patients clinical symptoms immediately after the head trauma (e.g. loss of conscience); clinical findings (mainly neurological status); imaging aspect (head CT scan).

Patients. We included 52 patients, 26 boys and 26 girls (sex ratio 1:1) diagnosed with epidural hematoma in our department on a 10 year period. The patients mean age was 14.5 months, ranging from 6 weeks to 3 years old. Most of the patients were 0-1 yo - $26(50 \%)$ patients, 12 (23\%) were 1-2 yo and $14(27 \%)$ aged 2-3. 
DOI: 10.2478/romneu-2014-0054

Radiological assessments. Every patient was examined at admission by CT scan or, sometimes, MRI. The neuroimaging examinations offer data regarding to the EDH location, associated brain lesions, associated skull lesions and their evolution in time (Figure 1 A, B).

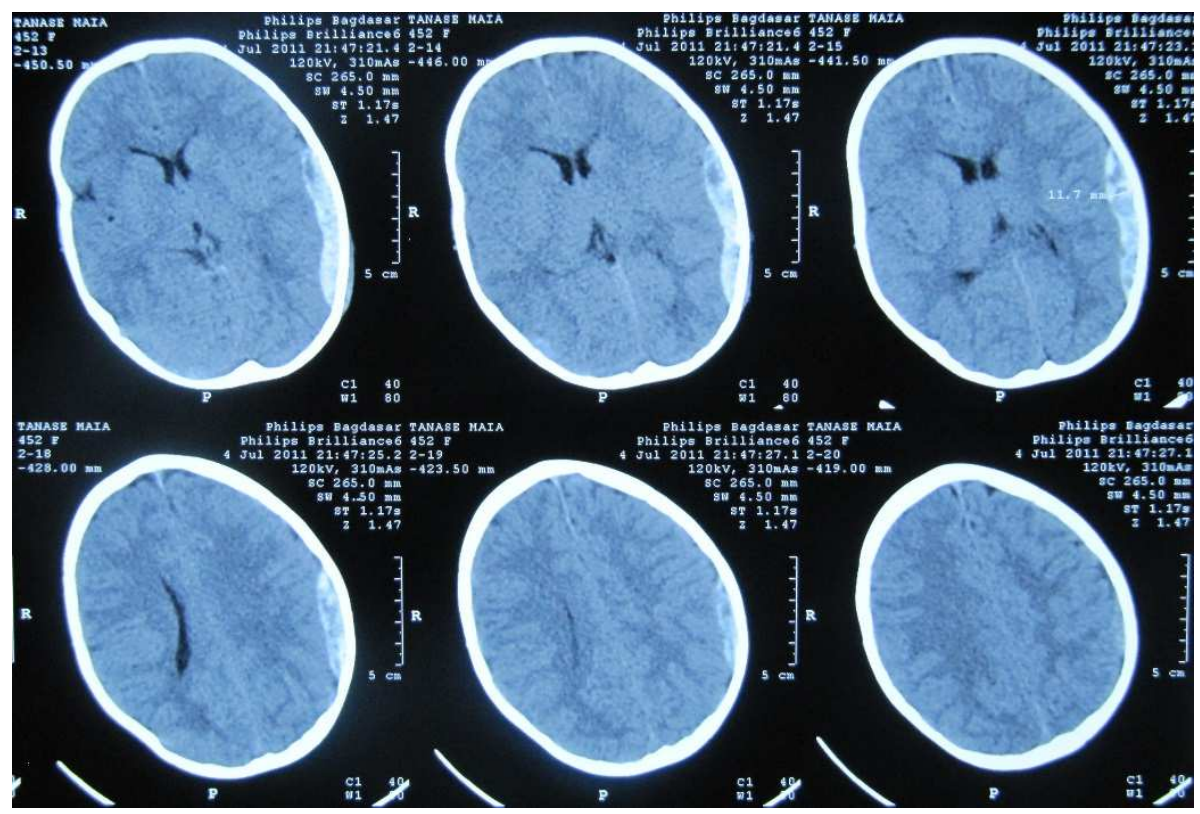

Figure $1 \mathrm{~A}$ - Acute subdural hematoma in a 3 yo child that suffered a moderate TBI by accidental falling from a different level. Clinical status: signs of intracranian hypertension, GCS=9points

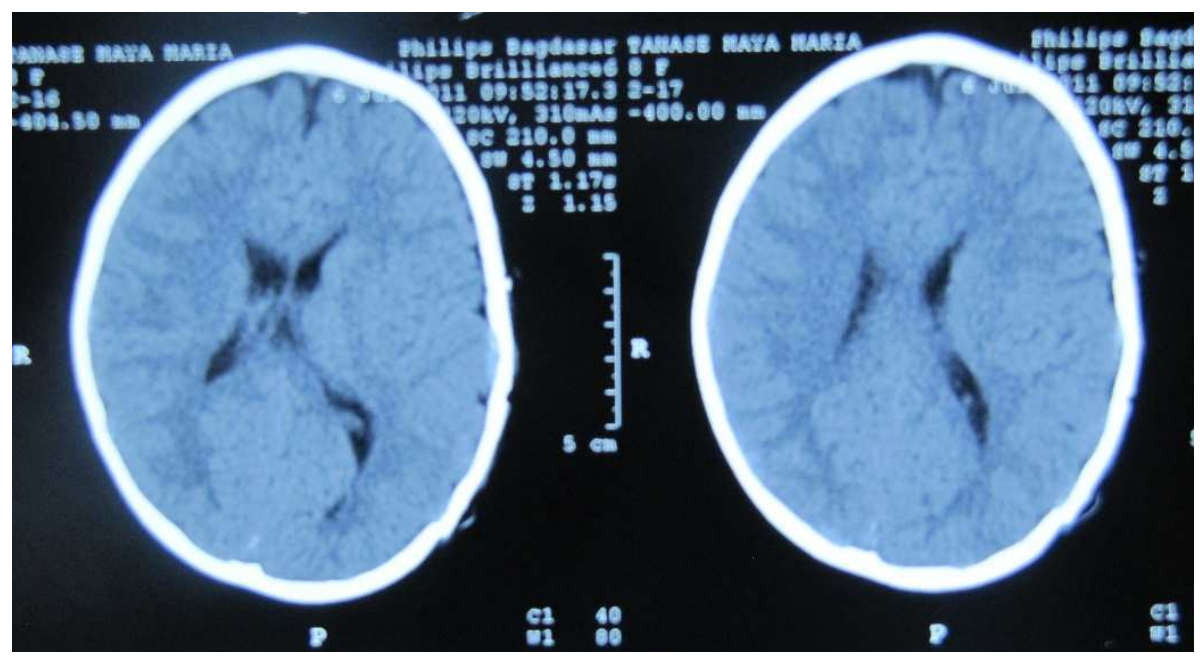

Figure 1 B - Postoperative aspect 
Treatment. 25 (48\%) patients were operated, while the other 27 were treated with symptomatic drugs and were carefully monitored clinically and by cerebral imaging. The surgical technique for EDH evacuation consisted in craniotomy based over the hematoma; it is imperious to obtain an adequate exposure of the hematoma, in order to control the bleeding source. After we lifted the bone flap, we removed the epidural hematoma by cup forceps, suction and irrigation, followed by the coagulation of the bleeding source. We've also incised the dura, to make sure there is no subdural bleeding subjacent to the epidural hematoma. The dura mater was then suspended to the perimeter of the craniotomy by several 'sleep stitches' and the bone flap was repositioned. For the posterior cranial fossa $\mathrm{EDH}$, the incision was performed on the midline, then we practiced a suboccipital bilateral craniotomy, followed by the evacuation of the hematoma and the tacking of the dura to the bone margins. We always placed a subgaleal drain tube maintained for 24 hours postoperatively.

Outcome evaluation. The clinical status was evaluated by Pediatric Glasgow Coma Scale at admission, neurological status during hospitalization and Glasgow Outcome Scale at discharge.

TABLE 1

Pediatric Glasgow Coma Scale and Glasgow Outcome Scale

\begin{tabular}{|c|c|c|}
\hline Pediatric Glasgow Coma Scale & & Glasgow Outcome Scale \\
\hline Eye opening & & $\mathrm{D}=$ dead \\
\hline spontaneous & 4 & PVS $=$ persistent vegetative state \\
\hline to speech & 3 & $\mathrm{SD}=$ severe disability \\
\hline to pain & 2 & MD-moderate disability \\
\hline no response & 1 & $\mathrm{GR}=$ good recovery \\
\hline \multicolumn{3}{|l|}{ Verbal response } \\
\hline $\begin{array}{l}\text { smiles, oriented to sounds, } \\
\text { follows objects, interacts }\end{array}$ & 5 & \\
\hline $\begin{array}{l}\text { cries but consolable, } \\
\text { inappropriate interactions }\end{array}$ & 4 & \\
\hline inconsistently inconsolable, moaning & 3 & \\
\hline inconsolable, agitated & 2 & \\
\hline no response & 1 & \\
\hline \multicolumn{3}{|l|}{ Motor response } \\
\hline moves spontaneously or purposefully & 6 & \\
\hline withdraws from touch & 5 & \\
\hline withdraws from pain & 4 & \\
\hline $\begin{array}{l}\text { abnormal flexion to pain for an } \\
\text { infant (decorticate response) }\end{array}$ & 3 & \\
\hline $\begin{array}{l}\text { extension to pain } \\
\quad \text { (decerebrate response) }\end{array}$ & 2 & \\
\hline no motor response & 1 & \\
\hline
\end{tabular}


DOI: 10.2478/romneu-2014-0054

\section{Results}

The most frequent cause for EDH was accidental falling, encountered in 38 (73.07\%) patients, $8(15.38 \%)$ patients couldn't specify the cause, $3(5.77 \%)$ patients suffered a blunt head trauma and 2 (3.84\%) patients were victims of road accidents (Table 2).

We classified the TBI based on the Glasgow Coma Scale, considering as mild TBI the patients with a GCS=13-15pts, moderate TBI at GCS $=12-9$ pts and severe TBI patients with GCS $=8$ or less. $39(75 \%)$ patients suffered a mild TBI, $7(13.46 \%)$ a moderate TBI and 6 (11.54\%) patients had a severe TBI.

The predominant clinical manifestations (Table 3) were those of intracranial hypertension such as headache, nausea/vomiting and drowsiness - 21 (40.30\%) patients and psychomotor agitation - 19 (36.53\%) patients. 8 (15.38\%) patients presented with drowsiness/decreased level of consciousness, $5(9.61 \%)$ had pupillary anomalies and $3(5.77 \%)$ patients presented with controlateral hemiparesis. The classical scenario with initial loss of consciousness followed by a lucid period and ulterior clinical manifestations was seen in $5(9.61 \%)$ patients.

TABLE 2

Head trauma mechanisms in our patients

\begin{tabular}{|c|c|c|}
\hline $\begin{array}{c}\text { Traumatic } \\
\text { mechanism }\end{array}$ & $\begin{array}{c}\text { No. of } \\
\text { patients }(\mathbf{n})\end{array}$ & Ratio (\%) \\
\hline accidental falling & 38 & 73.07 \\
\hline blunt head trauma & 3 & 5.77 \\
\hline road accident & 2 & 3.84 \\
\hline unspecified & 8 & 15.38 \\
\hline Other & 1 & 1.92 \\
\hline
\end{tabular}

5 (9.61\%) patients were comatose on admission. In 34 patients we found an epicranial hematoma and in 10 patients we had to evacuate the hematomas by puncturing.

Only 2 patients had a posterior fossa hematoma (Figure $2 \mathrm{~A}, \mathrm{~B}$ ), the other 50 patients presented with a supratentorial epidural blod clot. The most frequent localizations for of the epidural hematoma were parietal - 20 patients and temporalparietal - 11 patients.

A total of 37 patients presented a skull fracture subjacent to the epidural hematoma and in 34 patients we found an epicranial hematoma above. In 14 patients we had to evacuate the epicranial hematoma by puncturing. Other associated lesions were cerebral edema, subdural hematoma, subarachnoid hemorrhage and contusions (Table 4).

\section{TABLE 3}

Clinical manifestations

\begin{tabular}{|c|c|c|}
\hline Symptom & $\begin{array}{c}\text { No. of } \\
\text { patients (n) }\end{array}$ & Ratio (\%) \\
\hline Intracranial hypertension & 21 & 40.38 \\
\hline Psychomotor agitation & 19 & 36.53 \\
\hline $\begin{array}{c}\text { Drowsiness/decreased level } \\
\text { of counsciousness }\end{array}$ & 8 & 15.38 \\
\hline Coma (GCS<9pts) & 5 & 9.61 \\
\hline Nausea/vomiting & 5 & 9.61 \\
\hline Loss of counsciousness & 5 & 9.61 \\
\hline Pupilary anomalies & 4 & 7.7 \\
\hline Contralateral hemiparesis & 3 & 5.77 \\
\hline Headache & 3 & 5.77 \\
\hline Seizure & 1 & 1.92 \\
\hline
\end{tabular}




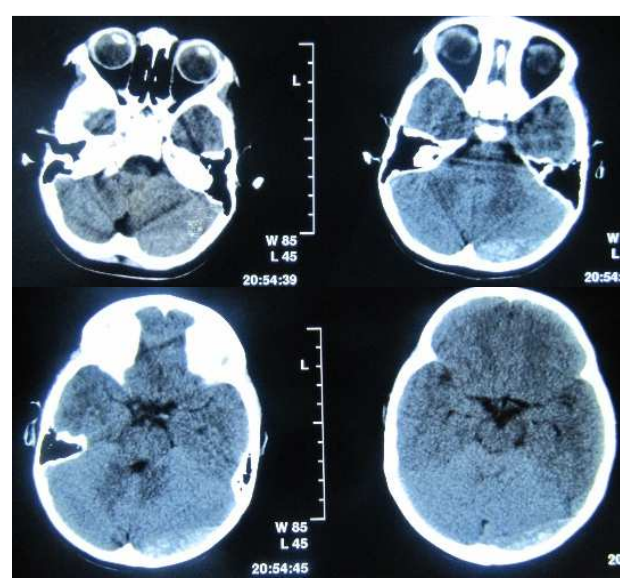

Figure $2 \mathrm{~A}$ - Posterior fossa EDH in a 2 yo child

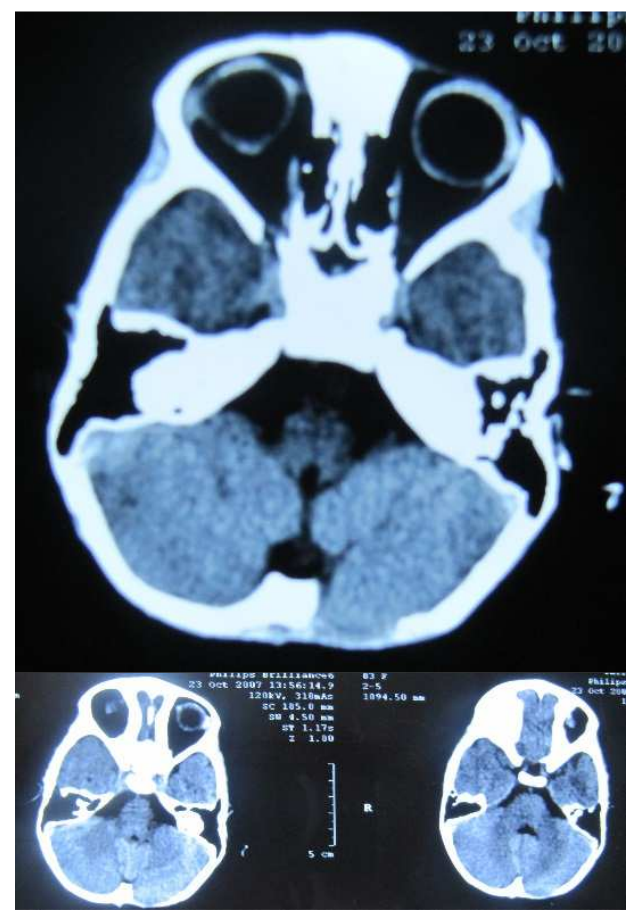

Figure $2 \mathrm{~B}$ - Postoperative aspect of the posterior cranian fossa EDH

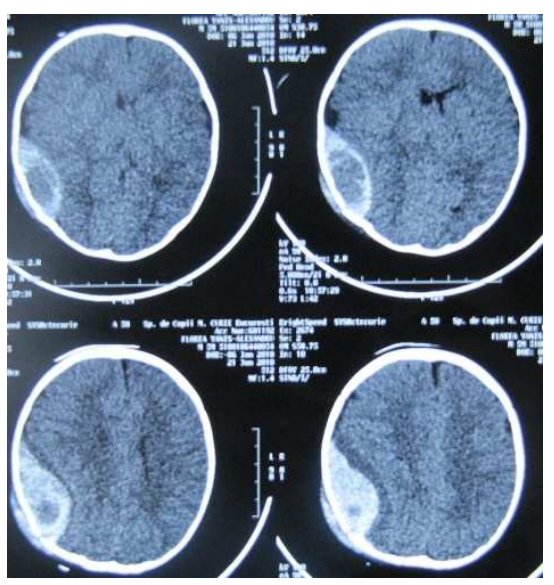

Figure $3 \mathrm{~A}$ - Temporal-parietal epidural hematoma in a 5 months baby that suffered a TBI caused by accidental falling from approximately 1 meter. GCS=9pts



Figure 3 B - Postoperative aspect

TABLE 4

Associated lesions

\begin{tabular}{|c|c|c|}
\hline Lesion type & $\begin{array}{c}\text { No. of } \\
\text { patients (n) }\end{array}$ & Ratio (\%) \\
\hline Skull fracture & 37 & 71.15 \\
\hline Epicranian hematoma & 34 & 65.38 \\
\hline Diffuse cerebral edema & 20 & 38.46 \\
\hline Subarachnoid hemorrhage & 13 & 25 \\
\hline Subdural hematoma & 6 & 11.54 \\
\hline Hemorrhagic contusion & 4 & 7.7 \\
\hline
\end{tabular}


DOI: 10.2478/romneu-2014-0054

25 (48\%) patients were operated, while the other 27 were treated with symptomatic drugs and were carefully monitored clinically and by cerebral imaging. In the nonoperated group, all the patients had a mild TBI, while in the surgically treated group of patients, 12 had a minor TBI, 7 a moderate TBI and 6 patients suffered a severe TBI. Clinically, the decision to surgically evacuate the hematoma was considered in case of over 2 points loss on Glasgow Coma Scale or the presence of pupillary changes. The imaging criteria for surgical treatment were: hematoma volume of minimum $25 \mathrm{~cm} 3$, epidural hyper density with a thickness larger than $10 \mathrm{~mm}$ and a mid-line shift (MLS) over $5 \mathrm{~mm}$ (Figure $3 \mathrm{~A}, \mathrm{~B}$ ).

$19(36.53 \%)$ of the operated patients presented postoperative complications, such as acute post-hemorrhagic anemia, pneumocephalus, headache or hematoma recurrence. 20 patients in the operated group were GOS/GR at discharge, 3 patients remained with neurological deficits postoperatively and 2 patients died; thus, the mortality rate in our study group was $3.84 \%$. Both deceased patients had severe associated traumatic injuries such as subdural hematomas, cerebral lacerations/contusions or moderate to severe thoracic/abdominal trauma, and the traumatic mechanism was road accidents.

All the nonoperated patients had a good clinical outcome, with the remission of the acute neurological symptoms (GOS/GR) at discharge. In 5 of the conservative treated patients the hematoma had a volume of over 25 $\mathrm{cm} 3$ and a midline shift of over $4 \mathrm{~mm}$, but the decision to not operate on them was based on the good neurological status (Figure 4 A, B).

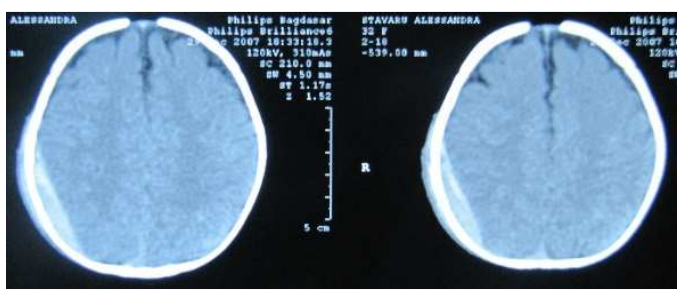

Figure $4 \mathrm{~A}$ - EDH in a 5 month girl, GCS=15pts

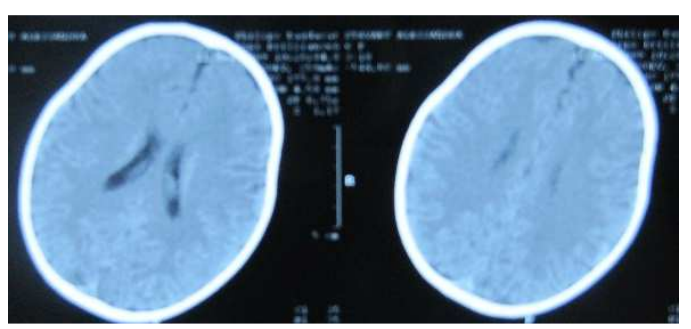

Figure 4 B - Control CT scan 2 weeks after the TBI

The medium number of hospitalization days was 9 for the operated patients (range 530 days) and 4 in the conservative treated patients.

\section{Discussions}

Our clinic is the most important pediatric neurosurgery center in the country, approaching a various cerebral pathology, mostly represented by traumatic head injuries. Our study includes all the patients diagnosed with an epidural hematoma, certified by cerebral CT scans, regardless the mechanism.

Cerebral CT scan is an essential diagnosis and monitoring element in neurosurgery, especially head trauma; it represents the imaging investigation of choice to diagnose intracerebral hematomas, and it offers details regarding the location of the hematoma, clot volume and thickness and the effect it has on the midline structures, ventricular system and basal cisterns. Therefore, a CT scan must be performed without delay $(11,12)$. All the 
patients included in our study were diagnosed and monitored by CT scan, and the imaging findings played an essential role in determining the type of treatment for each child.

Epidural hematomas were considered a neurosurgical emergency that can quickly lead to death of the patient if left untreated. Lately, the conservative treatment and careful monitoring of the clinical status and the neuroimaging aspect is considered a valid approach, on the condition that the patients neurological and imaging status allows it (10, 13). Our study comes to reinforce this concept that patients should not be treated surgically unless some well-defined clinical and imaging criteria are present. The criteria to decide in favor of surgical treatment considered were described, for adult population, by Bullock and colab. (11): hematoma volume larger than $30 \mathrm{~cm} 3$, midline shift of over $5 \mathrm{~mm}$ and clot thickness over $15 \mathrm{~mm}$ on cerebral CT scan (Figure 5 A, B, C, D). Also, Bullock and colab. (11) recommend that all comatose patients (GCS $<9$ pct) and pupillary asymmetry with an epidural hematoma should be operated in emergency to evacuate the blood clot.

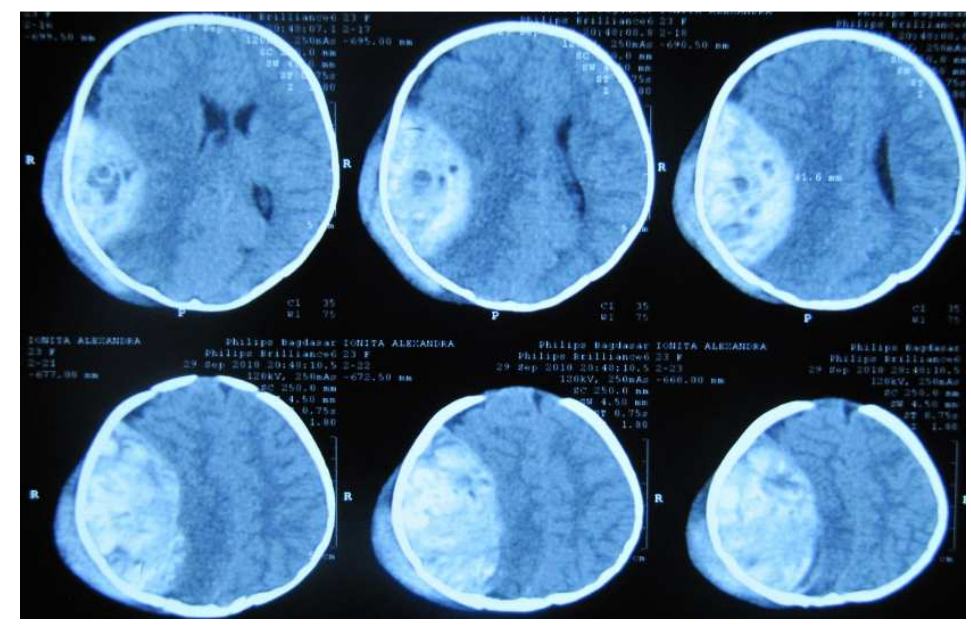

Figure $5 \mathrm{~A}$ - Acute EDH in a 4 month child, presenting with pupilarry anomalies, coma $(\mathrm{GCS}=5 \mathrm{pct})$

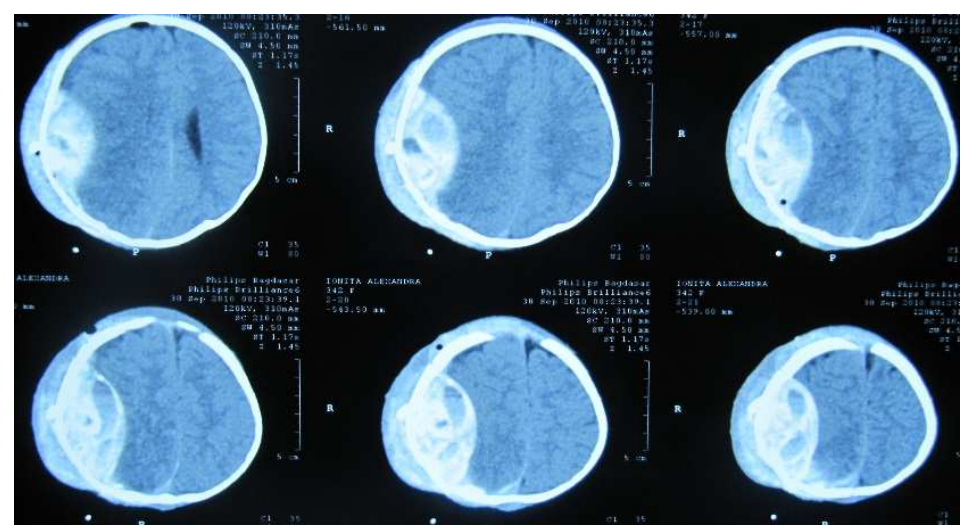

Figure 5 B - Recurrence of the EDH 


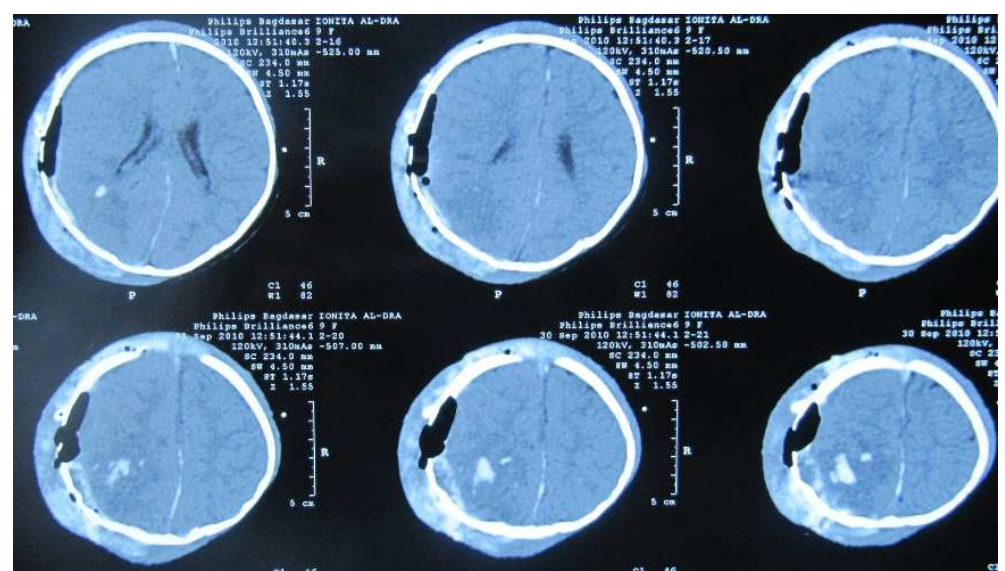

Figure 5 C - Postoperatively aspect



Figure 5 D - CT aspect 2 months postoperatively

The surgical treatment indications consider clinical status and imaging findings in each individual; patients included in our study were operated according to the following criteria, published in an earlier study $(11,14)$ :

- Comatose patient, pupillary anomalies and confirmation of diagnosis on CT scan require urgent surgery

- Coma and worsening of neurological state in case of EDH's volume $>25 \mathrm{ml}$

- EDH's volume > $30 \mathrm{ml}$, even in the absence of clinical signs
- EDH's > $25 \mathrm{ml}$, if EDH is located within the posterior fossa or temporal region

- Midline shift $>4 \mathrm{~mm}$, with worsening of clinical status

- EDH volume increase observed on neuroimaging monitoring.

Children that suffered head trauma but have no focal neurological deficits, with a CT scan appearance of an acute EDH having a volume $<25 \mathrm{ml}$, less than $10 \mathrm{~mm}$ thickness and MLS $<4 \mathrm{~mm}$ can receive conservative treatment, but with careful monitoring the 
clinical and neuroimaging status. These patients have to be admitted in a specialized clinic where they can quickly be operated in case of neurological worsening $(14,15)$.

Rivas and all. found that hematoma volume and severity of MLS were related to preoperative coma in patients with $\mathrm{EDH}(16)$. In comatose patients, a hematoma volume greater than $150 \mathrm{~cm} 3$ and an MLS greater than $12 \mathrm{~mm}$ were associated with increased mortality. Location of the lesion did not influence outcome. Selig et al. did not find a relationship between location of blood clot, MLS and outcome in 51 comatose patients undergoing surgery for EDH (17).

\section{Conclussions}

Both surgical treatment and conservative management of EDH have a good clinical outcome. Clinical and neuroimaging evaluation at admission/reevaluation plays an imperative role in deciding the appropriate therapeutic attitude for each patient. Conservative treatment should be applied in specialized centers that can assure quick measurements if the patients' neurological status worsens.

\section{Correspondence}

St.M. Iencean

"Grigore T. Popa" University of Medicine and

Pharmacy Iasi

E-mail:mirceasteffan@yahoo.com

\section{References}

1. Schmidek \& Sweet Operative Neurosurgical Techniques: indications, methods and results, 6th ed., vol. 2, Elsevier Saunders, PA, 2012

2. Choux M, Lena G, Genitori L. Intracranial hematomas.
In: Head Injuries in the Newborn and Infant, Raimondi A, Choux M, DiRocco C. (Eds), Springer-Verlag Inc, New York 1986. p.204.

3. Kim KA, Wang MY, Griffith PM, et al. Analysis of pediatric head injury from falls. Neurosurg Focus 2000; 8:e3.

4. Obana WG, Pitts LH. Management of head injury. Extracerebral lesions. Neurosurg Clin N Am 1991; 2:351. 5. Rocchi G, Caroli E, Raco A, et al. Traumatic epidural hematoma in children. J Child Neurol 2005; 20:569.

6. Aronyk KE. Post-traumatic hematomas. In: Pediatric Neurosurgery: Surgery of the Developing Nervous System, Cheek WR, Marlin AE, McLone DG, et al. (Eds), WB Mason, Philadelphia 1994. p.279.

7. Ciurea AV, Kapsalaki EZ, Coman TC, et al. Supratentorial epidural hematoma of traumatic etiology in infants. Childs Nerv Syst 2007; 23:335.

8. Schutzman SA, Barnes PD, Mantello M, Scott RM. Epidural hematomas in children. Ann Emerg Med 1993; 22:535.

9. Bullock et all, Guidelines for the Surgical Management of Traumatic Brain Injury, Neurosurgery March 2006, 3:58 10. Shu EBS, Leme RJA, Aguilar PH, Andrade AF, Teixeira MJ, Plese JPP. Traumatic acute giant epidural hematoma in a hydrocephalic shunted child. Pediatr Neurosurg 2000; 32: 176-179

11. Dachling Pang, M.D., F.R.C.S.(C), Joseph A. Horton, M.D., John M. Herron, PH.D., James E. Wilberger, JR., M.D., John K. VRIES, M.D. Nonsurgical management of extradural hematomas in children J Neurosurg 59:958971, 1983

12. Ciurea AV, Gorgan MR, Tascu A, Sandu AM, Rizea RE, Traumatic brain injury in infants and toddlers, 0-3 years old, J Med Life Vol 4, Is 3,July-September 2011

13. Balmer B, Boltshauser E, Altermatt S, Gobet R. Conservative management of significant epidural haematomas in children. Childs Nerv Syst 2006; 22: 363367

14. Rivas JJ, Lobato RD, Sarabia R, Cordobés F, Cabrera A, Gomez P Extradural hematoma: analysis of factors influencing the courses of 161 patients. Neurosurgery. 1988 Jul;23(1):44-51.

15. Seelig JM, Marshall LF, Toutant SM, Toole BM, Klauber MR, Bowers SA, Varnell JA. Traumatic acute epidural hematoma: unrecognized high lethality in comatose patients 